\title{
Older persons' experiences of adapting to daily life at home after hospital discharge: a qualitative metasummary
}

\author{
Christine Hillestad Hestevik $^{1^{*}}$ (D), Marianne Molin ${ }^{2,3}$, Jonas Debesay ${ }^{2}$, Astrid Bergland ${ }^{1}$ and Asta Bye $e^{2,4}$
}

\begin{abstract}
Background: Researchers have shown that hospitalisation can decrease older persons' ability to manage life at home after hospital discharge. Inadequate practices of discharge can be associated with adverse outcomes and an increased risk of readmission. This review systematically summarises qualitative findings portraying older persons' experiences adapting to daily life at home after hospital discharge.

Methods: A metasummary of qualitative findings using Sandelowski and Barroso's method. Data from 13 studies are included, following specific selection criteria, and categorised into four main themes.

Results: Four main themes emerged from the material: (1) Experiencing an insecure and unsafe transition, (2) settling into a new situation at home, (3) what would I do without my informal caregiver? and (4) experience of a paternalistic medical model.

Conclusions: The results emphasise the importance of assessment and planning, information and education, preparation of the home environment, the involvement of the older person and caregivers and supporting selfmanagement in the discharge and follow-up care processes at home. Better communication between older persons, hospital providers and home care providers is needed to improve the coordination of care and facilitate recovery at home. The organisational structure may need to be redefined and reorganised to secure continuity of care and the wellbeing of older persons in transitional care situations.
\end{abstract}

Keywords: Transition, Older people, Informal caregiver, Patient involvement, Communication, Qualitative research, Metasummary

\section{Background}

For older people, hospitalisation and changes in health status are often followed by feelings of stress, anxiety and uncertainty about the future [1]. Research has shown that hospitalisation decreases physical function, increases dependence [2] and decreases health-related quality of life (HRQOL). Older persons' HRQOL has also been found to decline during the post-discharge period $[3,4]$. After hospital discharge, these individuals tend to face many challenges to adjusting and coping with the possible repercussions of their illness (es) at home $[5,6]$.

\footnotetext{
*Correspondence: chhest@oslomet.no

${ }^{1}$ Department of Physiotherapy, Faculty of Health Sciences, OsloMet - Oslo

Metropolitan University, Oslo, Norway

Full list of author information is available at the end of the article
}

According to surveys, older people generally want to stay in their own home for as long as possible $[7,8]$. The policies of welfare states emphasise providing home care services with the goal of preserving the dignity and wellbeing of older people [7], and high-quality transitional care helps older people with multiple chronic conditions remain in their own homes for as long as possible. Additionally, it has the potential to minimise adverse events and rehospitalisation and increase the efficiency of the whole healthcare system $[9,10]$.

However, older persons face a myriad of challenges during this process. Multiple studies have reported that older persons experience a discontinuity of care on their way from hospital back into their community [11-14]. Shortened hospital stays and lack of continuity of care when older persons transition from hospital to home

(c) The Author(s). 2019 Open Access This article is distributed under the terms of the Creative Commons Attribution 4.0 International License (http://creativecommons.org/licenses/by/4.0/), which permits unrestricted use, distribution, and 
have been identified as serious challenges with negative implications, such as increased readmission rates and adverse medical events [15-17]. Furthermore, as Ekdahl et al. [18] stated, although older persons and geriatric syndromes are common in hospitals, these persons are commonly not prioritised, as healthcare professionals often perceive their cases as being too complex and time-consuming. Research shows that there is a lack of attention given to these persons' special needs and inadequate involvement of them and their families in their own care process $[12,19-21]$. Another problem is the inadequate communication of information between hospitals and other healthcare providers [22]. Bull et al. [23] found that the best predictors of older persons' satisfaction with discharge planning were a perception of continuity of care and preparedness to manage their own care. Almborg et al. [24] suggested that if the patients were provided with information about how to evaluate symptoms, manage medication and restrict activities, they felt more prepared after discharge. Moreover, the study emphasised that healthcare providers evaluation of the patient's needs after discharge is essential to the patient, and different professional disciplines should be involved depending on the patient's conditions and needs [25]. The older person's participation in the evaluation of their needs could be facilitated by asking them about problems in different areas of their life, among other strategies.

A key challenge of transitional care is providing healthcare adapted to the needs of older people-as perceived by themselves, not as defined by the professionals. Previous systematic reviews have found that transitional care interventions can be effective in improving outcomes [26-28]. However, challenges still remain in enhancing older persons' satisfaction with the healthcare services included in transitional care $[9,29]$. Knowledge about the experiences of older people regarding their own care is crucial to identifying and addressing issues related to the transition from hospital to home and may help reduce deficiencies and facilitate more satisfactory healthcare [30]. Thus, this metasummary aims to integrate current international findings in order to enhance the understanding of older persons' experiences of adapting to daily life at home after hospital discharge.

\section{Methods}

\section{Study design}

The techniques used to conduct this metasummary followed the methodological framework of Sandelowski and Barroso [31]. Qualitative metasummary is a quantitatively-oriented aggregation approach to research synthesis. Qualitative findings are collected from topical or thematic surveys of the data through a review of the relevant literature. In a qualitative metasummary, higher frequency findings are taken in order to find evidence of the repetition imperative to validity in quantitative research and to having discovered a pattern or theme.

\section{Study retrieval-search strategy}

With assistance from a librarian, the first author conducted a comprehensive literature review using five electronic databases (Medline, Embase, Academic Search Premier, Cinahl and PsycINFO). Hand searches were conducted and reference lists were examined. Keywords for the databases searches were: Aged, older patient, frail, elderly linked with patient discharge, patient transfer, patient handover, transitional care, hospital to home, hospital to municipal, hospital to community, patient (satisfaction, perception, experience, perspective, view) and interview or focus groups. The search was limited to studies published in the English language between 2006 and 2017 (current), aiming at findings that reflect patients' experiences of up-to-date healthcare systems.

\section{Selection criteria}

Titles, abstracts or full-text studies were scanned for adherence to the following inclusion criteria: studies using qualitative methods, a semi-structured or open-ended questioning approach; exploring older persons' self-reported experiences with relevance to the research topic; experiences of persons aged 65 or over adapting to life at home after hospital discharge. Original research, including peer-reviewed articles and doctoral theses, were included.

The studies were individually appraised using the Johanna Briggs Institute Qualitative Assessment and Review Instrument (JBI-QARI) [32]. The purpose of this appraisal was to assure that the reports met the inclusion criteria and to familiarise the authors with the informational content, methodological orientation and style and form of each study [31]. A cross-study comparative appraisal was also conducted using the GRADE-CERQual approach [33]. This method involves displaying the same key elements of information in each report alongside each other to determine how the studies related and help explain and contextualise the findings in the reports [31]. Individual and comparative appraisals were discussed among the authors until an agreement was reached.

\section{Synthesis of findings}

Data analysis in qualitative research consists of preparing and organising data (e.g. texts such as transcripts of interviews) for analysis and then reducing the data into broad patterns or themes [34]. The selected articles were reviewed, and relevant findings were extracted from each study, followed by grouping the findings into thematic statements and summarising these into abstracted themes. We calculated the frequency effect for each 
thematic statement by dividing the number of studies that mentioned a finding by the total number of studies included in the metasummary. This was done in order to quantify the strength of the findings, ensuring that the importance of these findings was neither neglected nor over-emphasised.

\section{Results}

The initial search identified 1345 studies. After removing duplicates, we ended up with 645 studies. An additional 625 studies were excluded after screening the titles and abstracts, as they lacked relevance to the study's topic. Twenty studies met inclusion criteria and were retrieved in full text for further analysis. Five studies were excluded because the findings were not relevant for the study's topic, as well as an additional two since some of the participants were interviewed in nursing homes, which made it difficult to separate findings concerning their experiences from nursing homes and their own homes. To make sure that no relevant studies were omitted, we scrutinised the reference lists of the selected studies and relevant literature reviews [35, 36], but no additional studies were found. No studies were excluded on the basis of quality appraisal (Table 1). One of the studies explored the perceptions of older persons who were readmitted to hospital within 28 days of discharge [37]. We decided to include this study, as it portrayed valuable experiences of the discharge process, as well as experiences of settling at home after discharge. The final sample included 13 studies meeting the criteria for the metasummary (Fig. 1). Each study was systematically assessed for its research question or statement of purpose, research method, sample size, participant characteristics (age, sex and diagnosis), setting and country in which the research took place (Table 2).

The samples for the individual studies ranged from 3 to 40 participants, each over 65 years old. The collective sample represented the experiences of 195 older adults, 95 women and 100 men. The 13 studies were conducted in 8 different countries. Eleven of the studies were conducted in the participants' homes after hospital discharge, one at a hospital follow-up visit within four weeks of discharge to home and one at the hospital after being readmitted to hospital following recent discharge to home. These studies addressed the clearly stated aims of the research, the data analysis was easy to follow, and the results were unambiguous throughout. The participants' voices were easily identifiable and separate from the researchers' own interpretations of the results. Furthermore, almost all of the studies included meaningful considerations of the relationship between the researcher and participants, and all addressed ethical issues.

The extraction phase resulted in 42 thematic statements (Table 3). During the abstraction phase, these themes were merged in order to capture the content of the findings accurately. Four main themes emerged from the material: (1) Experiencing an insecure and unsafe transition, (2) settling into a new situation at home, (3) what would I do without my informal caregiver? and (4) experience of a paternalistic medical model. Examples of participants' quotations illustrating these results are presented in (Additional file 1:Table S1).

\section{Theme 1: experiencing an insecure and unsafe transition} Many of the participants' experienced the transition home as insecure and, in some instances, unsafe and even dangerous [13-15, 30, 37-40]. This experience appears to be influenced by several factors, as reported under Theme 1 in Table 3. Several of the participants experienced a lack of information about their diagnosis, ongoing care and self-care at home, which led to feelings of anxiety and uncertainty [11, 13, 14, 30, 37, 38, 40-42]. Participants reported experiences of a rushed or poorly planned discharge, leading to information being omitted or given too hastily $[14,15,37,38,40-43]$. The participants had difficulties getting an overview of their medicine, as the name of the preparations and types of medicine were changed while they were in hospital $[5,14,15,30,38,40-42]$, and many participants said that no one talked to them about changes in their medication before discharge [14, 38, 41]. Several participants described a problem related to medication reconciliation $[14,38,40]$, meaning that their prescribed medicines did not match the medicines that should have been prescribed.

The discharge process was described by many of the participants as an anxious time because they were never quite sure when they were going to be allowed home [11, 14, 15, 42, 43]. Many participants experienced a lack of shared decision-making regarding discharge and ongoing care [11, 13, 14, 37, 40-42]. They also reported not understanding parts of the information received in the hospital [14, 37, 38, 40-42] and found that discharge information was not explained to them properly or well [14, 38, 40-42]. Even when healthcare personnel took the time to explain the information, participants did not always understand the explanations and information given to them [14, 37, 41]. Healthcare professionals' use of medical language and abbreviations, the busyness and stress of the situation and the older persons' inability to concentrate due to their medical condition seemed to affect their understanding of their own complex healthcare situations [14, 37, 38, 40-42]. The older persons' feelings of not being seen, heard or given an opportunity to take part in the care and planning had a negative impact on their experience of discharge and the transition to home [14, 40, 42]. 
Table 1 Quality assessment of the included studies using the JBI-QARI appraisal instrument

\begin{tabular}{|c|c|c|c|c|c|c|c|c|c|c|c|c|c|}
\hline Questions & $\begin{array}{l}\text { Andreasen } \\
\text { et al }\end{array}$ & $\begin{array}{l}\text { Bagge } \\
\text { et al. }\end{array}$ & $\begin{array}{l}\text { Dilworth } \\
\text { et al. }\end{array}$ & $\begin{array}{l}\text { Dossa } \\
\text { et al. }\end{array}$ & Jones & $\begin{array}{l}\text { Karlsson } \\
\text { et al. }\end{array}$ & $\begin{array}{l}\text { Knight } \\
\text { et al. }\end{array}$ & $\begin{array}{l}\text { McKeown } \\
\text { et al }\end{array}$ & $\begin{array}{l}\text { Neiterman } \\
\text { et al. }\end{array}$ & $\begin{array}{l}\text { Perry } \\
\text { et al. }\end{array}$ & $\begin{array}{l}\text { Reay } \\
\text { et al. }\end{array}$ & $\begin{array}{l}\text { Rydeman } \\
\text { et al. }\end{array}$ & $\begin{array}{l}\text { Slatyer } \\
\text { et al. }\end{array}$ \\
\hline $\begin{array}{l}\text { Is there congruity } \\
\text { between the stated } \\
\text { philosophical } \\
\text { perspective and the } \\
\text { research } \\
\text { methodology? }\end{array}$ & U & U & NA & U & $Y$ & $Y$ & U & $Y$ & U & Y & Y & NA & Y \\
\hline $\begin{array}{l}\text { Is there congruity } \\
\text { between the } \\
\text { research } \\
\text { methodology and } \\
\text { the research } \\
\text { question or } \\
\text { objectives? }\end{array}$ & Y & Y & Y & Y & Y & Y & Y & Y & Y & Y & Y & Y & Y \\
\hline $\begin{array}{l}\text { Is there congruity } \\
\text { between the } \\
\text { research } \\
\text { methodology and } \\
\text { the methods used } \\
\text { to collect data? }\end{array}$ & Y & Y & Y & Y & Y & U & Y & Y & Y & Y & Y & Y & Y \\
\hline $\begin{array}{l}\text { Is there congruity } \\
\text { between the } \\
\text { research } \\
\text { methodology and } \\
\text { the representation } \\
\text { and analysis of } \\
\text { data? }\end{array}$ & Y & Y & Y & Y & Y & Y & Y & Y & Y & Y & Y & Y & Y \\
\hline $\begin{array}{l}\text { Is there congruity } \\
\text { between the } \\
\text { research } \\
\text { methodology and } \\
\text { the interpretation } \\
\text { of results? }\end{array}$ & Y & Y & Y & Y & Y & Y & $\mathrm{N}$ & Y & Y & Y & Y & Y & Y \\
\hline $\begin{array}{l}\text { Is there a } \\
\text { statement that } \\
\text { locates the } \\
\text { researcher culturally } \\
\text { or theoretically? }\end{array}$ & $N$ & $\mathrm{~N}$ & U & $\mathrm{N}$ & Y & $\mathrm{N}$ & $\mathrm{N}$ & Y & $\mathrm{N}$ & Y & $\mathrm{N}$ & $\mathrm{N}$ & $\mathrm{N}$ \\
\hline $\begin{array}{l}\text { Is the influence of } \\
\text { the researcher on } \\
\text { the research, and } \\
\text { vice versa, } \\
\text { addressed? }\end{array}$ & Y & Y & Y & Y & Y & Y & N & Y & N & $N$ & U & Y & Y \\
\hline $\begin{array}{l}\text { Are participants, } \\
\text { and their voices, } \\
\text { adequately } \\
\text { represented? }\end{array}$ & Y & Y & Y & Y & Y & Y & U & Y & Y & Y & Y & Y & Y \\
\hline $\begin{array}{l}\text { Is the research } \\
\text { ethical according } \\
\text { to current criteria } \\
\text { or, for recent } \\
\text { studies, is there } \\
\text { evidence of ethical } \\
\text { approval by an } \\
\text { appropriate body? }\end{array}$ & Y & Y & Y & Y & Y & Y & Y & Y & Y & Y & Y & Y & Y \\
\hline $\begin{array}{l}\text { Do the conclusions } \\
\text { drawn in the } \\
\text { research report } \\
\text { flow from the } \\
\text { analysis, or } \\
\text { interpretation, of } \\
\text { the data? }\end{array}$ & Y & Y & Y & Y & Y & Y & Y & Y & Y & Y & Y & Y & Y \\
\hline
\end{tabular}




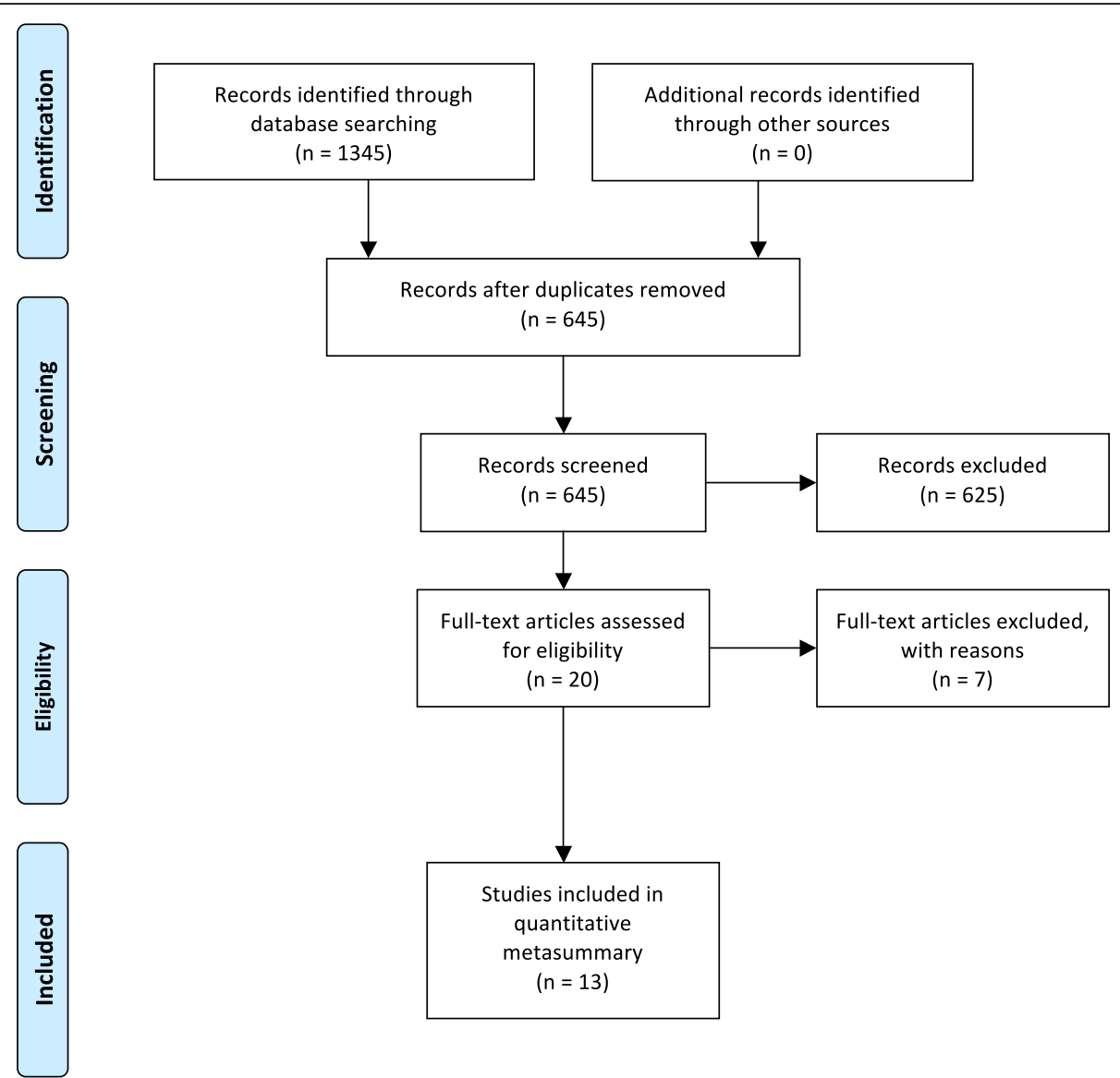

Fig. 1 Identification and selection of studies. Source: Moher, Liberati, Tetzlaff, Altman, and The PRISMA Group. (2009). Note. For more information, visit www.prisma-statement.org

Participants also described several examples of errors in the treatment that were either because of, or made worse by, poor communication between themselves and their caregivers and between healthcare providers $[13,14,30$, $38,40,41]$. Having several caregivers responsible for organising care seemed to lead to even more confusion and discontinuity of care $[13-15,37,38,40]$, and some of the participants experienced conflicting opinions about their treatment and care between the different health professionals overseeing their case $[13,14,38,40]$. Better communication between staff, older persons and their caregivers could, therefore, significantly improve the older persons' experience of the discharge procedure $[14,37$, 42], as described by Rydeman et al. [42]:

The participants' individual needs were satisfied when professionals were perceived as being knowledgeable and committed in their caring functions. They gave, for instance, comprehensible and individually adjusted information, instructions and explanations regarding the disease and treatment, the likely disease progress and the discharge time scale. All written information was highly legible, e.g. typewritten with upper-case letters. The professionals showed respect, were attentive to any emotional impact, and the older persons' and their relatives' points of view were considered.

When these needs were satisfied, the older persons experienced a well-prepared and timely discharge, resulting in a harmonious feeling and a sense of readiness to return to daily life at home [30, 42, 43].

\section{Theme 2: settling into a new situation at home}

Factors that influenced the experience of settling into a new situation at home are reported under Theme 2 in Table 3. Numerous studies reported that the participants were keen to return home to the security of their own environment, which was associated with recovery, independence and personal control [11, 15, 37-39, 43]. However, adaptation to daily life after discharge from the hospital was seen by many of the participants as a real challenge $[5,13,15,30,38-40,43]$, as cooking, dressing, bathing and other daily activities were difficult to manage immediately after discharge $[5,11,15,30,38,39,43]$. Health problems, such as tiredness, pain and lack of appetite, also caused distress [5, 30, 43]. 
Table 2 Characteristics of the qualitative studies selected for analysis

\begin{tabular}{|c|c|c|c|c|c|}
\hline $\begin{array}{l}\text { Author, year, } \\
\text { country }\end{array}$ & Country & $\begin{array}{l}\text { Data collection/ } \\
\text { methodology }\end{array}$ & Sample & Setting & Cause of admission (n) \\
\hline $\begin{array}{l}\text { Andreasen, J, et al. } \\
\text { (2015) } \\
{[30]}\end{array}$ & Denmark & $\begin{array}{l}\text { Semi-structured interviews/ } \\
\text { interpretive description }\end{array}$ & $\begin{array}{l}7 \text { Women } \\
7 \text { Men } \\
\text { Age } \\
\text { range: } \\
\text { 69-93 } \\
\text { Mean } \\
\text { age: } 80.6 \\
\text { years }\end{array}$ & $\begin{array}{l}\text { At home approx. } 1 \text { week after } \\
\text { discharge from hospital }\end{array}$ & $\begin{array}{l}4 \text { Pneumonia } \\
1 \text { Emboli } \\
1 \text { Amputee } \\
1 \text { Fall } \\
1 \text { Brain abscess } \\
1 \text { Weight loss } \\
1 \text { Hypoglycaemia } \\
1 \text { Renal failure } \\
1 \text { Pancreatitis } \\
1 \text { Type } 2 \text { diabetes } \\
1 \text { Dizziness }\end{array}$ \\
\hline $\begin{array}{l}\text { Bagge et al. } \\
\text { (2014) } \\
{[41]}\end{array}$ & $\begin{array}{l}\text { New } \\
\text { Zealand }\end{array}$ & $\begin{array}{l}\text { Semi-structured interviews/ } \\
\text { thematic analysis }\end{array}$ & $\begin{array}{l}21 \\
\text { Women } \\
19 \text { Men } \\
\text { Age } \\
\text { range: } \\
75-89 \\
\text { Mean } \\
\text { age: not } \\
\text { stated }\end{array}$ & $\begin{array}{l}\text { At home } 1-2 \text { weeks after } \\
\text { discharge from hospital }\end{array}$ & Not reported \\
\hline $\begin{array}{l}\text { Dilworth et al. } \\
(2012) \\
{[40]}\end{array}$ & Australia & $\begin{array}{l}\text { Semi-structured interview/ } \\
\text { thematic analysis }\end{array}$ & $\begin{array}{l}1 \text { Woman } \\
2 \\
\text { MenAge } \\
\text { range: } \\
\text { not } \\
\text { stated } \\
\text { Mean } \\
\text { age: not } \\
\text { stated }\end{array}$ & $\begin{array}{l}\text { In hospital after being } \\
\text { readmitted to hospital } \\
\text { following recent discharge } \\
\text { (within } 28 \text { days) to their homes }\end{array}$ & $\begin{array}{l}1 \text { Renal failure/Fall } \\
1 \text { Cellulitis/Pulmonary emboli } \\
1 \text { Dizziness }\end{array}$ \\
\hline $\begin{array}{l}\text { Dossa et al. (2012) } \\
\text { [13] }\end{array}$ & $\begin{array}{l}\text { United } \\
\text { States }\end{array}$ & $\begin{array}{l}\text { Semi-structured interviews/ } \\
\text { thematic coding technique } \\
\text { informed by grounded } \\
\text { theory methodology }\end{array}$ & $\begin{array}{l}18 \text { Men } \\
\text { Age } \\
\text { range:78- } \\
88 \\
\text { Mean } \\
\text { age: not } \\
\text { stated }\end{array}$ & $\begin{array}{l}\text { At home } 2 \text { weeks, } 1 \text { month } \\
\text { and } 2 \text { months after discharge } \\
\text { from hospital }\end{array}$ & $\begin{array}{l}\text { The discharge diagnoses included total hip } \\
\text { or total knee replacements, laminectomy, } \\
\text { diabetes, arthritis, coronary artery disease, } \\
\text { hypertension and alcohol abuse }\end{array}$ \\
\hline $\begin{array}{l}\text { Jones, GB (2012) } \\
\text { [38] }\end{array}$ & $\begin{array}{l}\text { United } \\
\text { States }\end{array}$ & $\begin{array}{l}\text { Semi-structured interviews/ } \\
\text { phenomenological } \\
\text { hermeneutical } \\
\text { interpretation method }\end{array}$ & $\begin{array}{l}16 \\
\text { Women } \\
4 \text { Men } \\
\text { Age } \\
\text { range: } \\
65-89 \\
\text { Mean } \\
\text { age: } 75 \\
\text { years }\end{array}$ & $\begin{array}{l}\text { At follow-up visit in two } \\
\text { cardiology/cardiovascular } \\
\text { clinics within } 4 \text { weeks of } \\
\text { discharge from hospital }\end{array}$ & $\begin{array}{l}\text { The majority of participants had a } \\
\text { cardiovascular medical diagnosis ( } n=12 \text {, } \\
60 \% \text { ) or experienced cardiovascular surgical } \\
\text { procedures }(n=8,40 \%)\end{array}$ \\
\hline $\begin{array}{l}\text { Karlsson et al. } \\
(2016) \\
{[43]}\end{array}$ & Sweden & $\begin{array}{l}\text { Qualitative interview/ } \\
\text { content analysis }\end{array}$ & $\begin{array}{l}7 \text { Women } \\
8 \text { Men } \\
\text { Age } \\
\text { range: } \\
65-86 \\
\text { Mean } \\
\text { age: } 71 \\
\text { years }\end{array}$ & $\begin{array}{l}\text { At home within } 2 \text { months } \\
\text { after discharge from hospital }\end{array}$ & $\begin{array}{l}7 \text { Surgery of aortic aneurysm } \\
1 \text { Epiglottitis } \\
1 \text { Pneumonia, sepsis } \\
1 \text { Pneumonia } \\
1 \text { Pulmonary edema } \\
1 \text { Pneumonia, sepsis, kidney failure } \\
1 \text { Myasthenia gravis, cardiac arrest } \\
1 \text { Unknown } \\
1 \text { Allergic shock }\end{array}$ \\
\hline $\begin{array}{l}\text { Knight et al. } \\
\text { (2011) } \\
{[14]}\end{array}$ & $\begin{array}{l}\text { United } \\
\text { Kingdom }\end{array}$ & $\begin{array}{l}\text { Semi-structured interviews/ } \\
\text { thematic analysis }\end{array}$ & $\begin{array}{l}4 \text { Women } \\
3 \text { Men } \\
\text { Age } \\
\text { range: } \\
75-91 \\
\text { Mean } \\
\text { age: } 82.6 \\
\text { years }\end{array}$ & $\begin{array}{l}\text { At home } 6 \text { weeks to } 3 \text { months } \\
\text { after discharge from hospital }\end{array}$ & Not reported \\
\hline Mckeown et al. & Ireland & Qualitative interviews/ & 5 Women & At home 2 weeks after & Not reported \\
\hline
\end{tabular}


Table 2 Characteristics of the qualitative studies selected for analysis (Continued)

\begin{tabular}{|c|c|c|c|c|c|}
\hline $\begin{array}{l}\text { Author, year, } \\
\text { country }\end{array}$ & Country & $\begin{array}{l}\text { Data collection/ } \\
\text { methodology }\end{array}$ & Sample & Setting & Cause of admission (n) \\
\hline $\begin{array}{l}(2007) \\
{[5]}\end{array}$ & & $\begin{array}{l}\text { phenomenological } \\
\text { approach }\end{array}$ & $\begin{array}{l}6 \text { Men } \\
\text { Age } \\
\text { range: } \\
71-92 \\
\text { Mean } \\
\text { age: } 81 \\
\text { years }\end{array}$ & discharge from hospital & \\
\hline $\begin{array}{l}\text { Neitherman et al. } \\
\text { (2015) } \\
{[15]}\end{array}$ & Canada & $\begin{array}{l}\text { Semi-structured interviews/ } \\
\text { thematic analysis }\end{array}$ & $\begin{array}{l}7 \text { Women } \\
10 \text { Men } \\
\text { Age } \\
\text { range: } \\
70-89 \\
\text { Mean } \\
\text { age: } 79 \\
\text { years }\end{array}$ & $\begin{array}{l}\text { At home } 2-5 \text { weeks after } \\
\text { discharge } \\
\text { from hospital }\end{array}$ & $\begin{array}{l}\text { The most common diagnoses for } \\
\text { hospitalisation were cardiovascular } \\
\text { conditions (congestive heart failure, stroke) } \\
\text { and respiratory problems (chronic } \\
\text { obstructive pulmonary disease, pneumonia). } \\
\text { Other patients had a variety of health } \\
\text { problems, including diabetes, kidney disease, } \\
\text { gastro-intestinal and neurological problems } \\
\text { and cancer }\end{array}$ \\
\hline $\begin{array}{l}\text { Perry et al. (2011) } \\
\text { [11] }\end{array}$ & $\begin{array}{l}\text { New } \\
\text { Zealand }\end{array}$ & $\begin{array}{l}\text { Semi-structured interviews/ } \\
\text { interpretative } \\
\text { phenomenological analysis }\end{array}$ & $\begin{array}{l}8 \text { Women } \\
3 \text { Men } \\
\text { Age } \\
\text { range: } \\
66-88 \\
\text { Mean } \\
\text { age } 76.3 \\
\text { years }\end{array}$ & $\begin{array}{l}\text { At home approx. } 6 \text { weeks after } \\
\text { discharge from hospital }\end{array}$ & Orthopaedic lower limb surgery \\
\hline $\begin{array}{l}\text { Reay et al. (2015) } \\
\text { [39] }\end{array}$ & Australia & $\begin{array}{l}\text { Semi-structured interviews } \\
\text { /Giorgi's } \\
\text { phenomenological } \\
\text { method }\end{array}$ & $\begin{array}{l}6 \text { Women } \\
4 \text { Men } \\
\text { Age } \\
\text { range: } \\
\text { not } \\
\text { stated } \\
\text { Mean } \\
\text { age: not } \\
\text { stated }\end{array}$ & $\begin{array}{l}\text { At home approx. } 3 \text { weeks } \\
\text { after discharge }\end{array}$ & Total hip replacement surgery \\
\hline $\begin{array}{l}\text { Rydeman et al. } \\
(2008) \\
{[42]}\end{array}$ & Sweden & $\begin{array}{l}\text { Semi-structured interviews/ } \\
\text { grounded theory }\end{array}$ & $\begin{array}{l}7 \text { Women } \\
10 \text { Men } \\
\text { Age } \\
\text { range: } \\
65-91 \\
\text { Mean } \\
\text { age: } 79 \\
\text { years }\end{array}$ & $\begin{array}{l}\text { At home 4-8 weeks after } \\
\text { discharge from hospital }\end{array}$ & $\begin{array}{l}4 \text { Infection } \\
4 \text { Heart problems } \\
1 \text { Rheumatic disease } \\
3 \text { Intestinal problems } \\
1 \text { Dehydration } \\
1 \text { Fracture } \\
1 \text { Pneumonia stroke } \\
1 \text { Intoxication }\end{array}$ \\
\hline $\begin{array}{l}\text { Slatyer et al. } \\
\text { (2013) } \\
{[37]}\end{array}$ & Australia & $\begin{array}{l}\text { Semi-structured interviews/ } \\
\text { thematic content analysis }\end{array}$ & $\begin{array}{l}6 \text { Women } \\
6 \text { Men } \\
\text { Age } \\
\text { range: } \\
72-91 \\
\text { Mean } \\
\text { age: } 81.6 \\
\text { years }\end{array}$ & $\begin{array}{l}\text { At home within } 28 \text { days of } \\
\text { discharge (after readmission } \\
\text { to hospital) }\end{array}$ & $\begin{array}{l}\text { Breathing, gastric, renal or cardiovascular } \\
\text { problems; falls; or chest pain } 7\end{array}$ \\
\hline
\end{tabular}

Many participants returned to a home environment that was not ready or appropriate for their new health situation $[5,13,15,30,38-40]$. Environmental challenges in the home posed significant activity impediments and could result in them resorting to unsafe practices $[5,13,30,38,39]$. Many participants also experienced lack of specialized equipment and supplies necessary for managing at home, such as walkers, adapted toilets, shower chairs, scales, glucose meters, etc. $[5,13$, 30, 38, 39]. They reported that the healthcare provided by home care was not suited to their individual needs.
The participants often did not get the right type of care and/or help at the right time of day or even the right day of the week, when they needed it most $[5,15,30$, $38-40,43]$. When different people from the care services visited at unexpected times, it was disturbing to the older persons' effort to get back to their daily routines after discharge [15, 30].

Participants also reported not being capable of participating in meaningful activities anymore, primarily due to their physical condition, leading to a more isolated social life $[5,15,30,39,43]$. A loss in social life created 
Table 3 Findings, including main themes and thematic statements, with calculated frequency effect \% (rounded to nearest whole number)

\begin{tabular}{|c|c|c|}
\hline Findings & Included studies & Frequency effect \% \\
\hline \multicolumn{3}{|l|}{ Theme 1: Experiencing an Insecure and Unsafe Transition } \\
\hline Lack of information about health situation, treatment and/or care & {$[11,13,14,30,37,38,40-42]$} & $69 \%$ \\
\hline Experience of rushed discharge & {$[14,15,37,38,40-43]$} & $62 \%$ \\
\hline Confusion about medication & {$[5,14,15,30,38,40-42]$} & $62 \%$ \\
\hline Lack of involvement in own treatment and care & {$[11,13,14,37,40-42]$} & $54 \%$ \\
\hline Not being involved in decisions about own life & {$[11,13,14,37,40-42]$} & $54 \%$ \\
\hline Not understanding information & {$[14,37,38,40-42]$} & $46 \%$ \\
\hline Several providers coordinating care led to discontinuity of care & {$[13-15,37,38,40]$} & $46 \%$ \\
\hline Errors in treatment & {$[13,14,30,38,40,41]$} & $46 \%$ \\
\hline Discharge information not explained well & {$[14,38,40-42]$} & $38 \%$ \\
\hline Lack of information about when to go home & {$[11,14,15,42,43]$} & $38 \%$ \\
\hline Lack of communication between the different service providers & {$[13,14,30,38,40]$} & $38 \%$ \\
\hline Conflicting opinions between healthcare providers & {$[13,14,38,40]$} & $31 \%$ \\
\hline Lack of medical reconciliation & {$[14,38,40]$} & $23 \%$ \\
\hline Experience of well-prepared and timely discharge & {$[30,42,43]$} & $23 \%$ \\
\hline \multicolumn{3}{|l|}{ Theme 2: Settling into a New Situation at Home } \\
\hline Dependent on additional help from others & {$[5,11,13-15,30,37-43]$} & $100 \%$ \\
\hline Losing independence & {$[11,13,15,30,38-43]$} & $77 \%$ \\
\hline Finding the transition back home a challenge & {$[5,13,15,30,38-40,43]$} & $62 \%$ \\
\hline Home not being prepared & {$[5,13,15,30,38-40,42]$} & $54 \%$ \\
\hline Problems performing daily activities & {$[5,11,15,30,38,39,43]$} & $54 \%$ \\
\hline Not receiving care according to needs & {$[5,15,30,38-40,43]$} & $54 \%$ \\
\hline Wanting to maintain and regain independence & {$[11,15,37-39,43]$} & $46 \%$ \\
\hline Not feeling ready to go home & {$[11,37,40,42,43]$} & $38 \%$ \\
\hline Feeling confident to go home & {$[11,37,39,42,43]$} & $38 \%$ \\
\hline Not being able to participate in meaningful activities & {$[5,15,30,39,43]$} & $38 \%$ \\
\hline Feeling lonely and isolated & {$[5,15,30,39,43]$} & $38 \%$ \\
\hline Lack of specialised equipment & {$[5,13,30,38,39]$} & $38 \%$ \\
\hline Changing healthcare personnel disturbed effort to get back to daily routines & {$[15,30]$} & $15 \%$ \\
\hline Feeling depressed & {$[15,30]$} & $15 \%$ \\
\hline Experiencing no meaning in life & {$[15,30]$} & $15 \%$ \\
\hline Wanting to die & {$[15,30]$} & $15 \%$ \\
\hline \multicolumn{3}{|l|}{ Theme 3: What Would I do Without My Informal Caregiver? } \\
\hline Dependent on informal caregivers for medication and healthcare & {$[5,14,15,37,38,40-42]$} & $62 \%$ \\
\hline Dependent on family and friends to manage daily activities at home & {$[5,11,15,30,37-39,43]$} & $62 \%$ \\
\hline Being aware of the effort put in by informal caregivers & {$[5,11,30,39,40]$} & $38 \%$ \\
\hline Importance of strong, positive relationships with family and friends & {$[5,11,15,30,39]$} & $38 \%$ \\
\hline Dependent on informal caregivers to understand information & {$[14,37,41,42]$} & $31 \%$ \\
\hline Illness putting a strain on relationship with family and friends & {$[11,30,39]$} & $23 \%$ \\
\hline Feeling like a burden & {$[11,30,39]$} & $23 \%$ \\
\hline \multicolumn{3}{|l|}{ Theme 4: Experience of a Paternalistic Model } \\
\hline Healthcare personnel perceived as distant and stressed & {$[11,13,14,30,37,38,41,42]$} & $62 \%$ \\
\hline Not being seen or heard & {$[11,13,14,40-42]$} & $46 \%$ \\
\hline
\end{tabular}


Table 3 Findings, including main themes and thematic statements, with calculated frequency effect \% (rounded to nearest whole number) (Continued)

\begin{tabular}{lll}
\hline Findings & Included studies & Frequency effect \% \\
\hline Reluctant to ask & {$[14,37,40-42]$} & $38 \%$ \\
Healthcare personnel perceived as authoritarian & {$[11,41-43]$} & $31 \%$ \\
Doctor knows best & {$[14,40-42]$} & $31 \%$ \\
\hline
\end{tabular}

negative consequences, such as loneliness, depression, a feeling of having no one to exist for and even, for some, a wish to die $[15,30]$.

\section{Theme 3: what would I do without my informal caregiver?}

Following discharge, many of the participants reported that they were dependent on additional assistance, usually provided by an informal caregiver, ranging from a spouse to an adult child, friends or neighbours [5, 11, 13-15, 30, 37-43]. The experiences related to this theme are listed under Theme 3 in Table 3. Personal networks and social support seemed to be a crucial factor for a successful recovery for most of the participants. Caregiver support included medication and care management, cooking, cleaning, dressing, shopping, transportation, personal hygiene, incision care and dressing changes and symptom management $[5,14,15,38-41,43]$. Some participants needed walkers and/or other assistance devices, and in some cases these arrangements had to be made by informal caregivers [15, 38].

Strong, positive relationships with a spouse, family, friends and/or neighbours were emphasised as being important factors in the daily life for older patients $[5,11,15$, 30, 39]. However, they were worried that their illness would put a strain on these relationships [11, 30, 39]. The participants were aware that their informal caregivers had limited time due to other commitments, and they did not want to overburden the caregivers $[5,11,30,39]$. Several of them reported a feeling of being a burden to their closest relatives, resulting in feelings of stress, anxiety and guilt $[11,30,39]$.

\section{Theme 4: experience of a paternalistic medical model}

The paternalistic model describes the older person's compliance with medical authority, and this was apparent in some of the participants' experiences during their stay in hospital and under follow-up care. Factors contributing to this experience are listed under Theme 4 in Table 3. In general, the participants trusted the system, did what they were told and had no complaints [11, 37, 40, 41]. They seemed to rely on and accept the decisions and assessments made by physicians and nurses because they were regarded as being authoritarian or that 'they know best' $[11,40-43]$, making them reluctant to critically question staff about their treatment and care [14, 37, 40-42]. They experienced healthcare staff who were stressed, distant or in a hurry and did not have the time to talk to them [11, $13,14,30,37,38,41,42]$. Some participants equated asking questions as arguing with the healthcare staff [41], and some felt they were not being heard when they questioned decisions made by the doctors [14, 38, 40, 42]. Some also felt patronised by the health professionals [11]. In situations where needs were not met after discharge, the older persons felt treated as objects and insistent and tiresome cases, and this had negative consequences for the person's wellbeing [30, 42].

\section{Discussion}

Our analyses of these studies indicate that during hospital discharge and transitional care, older persons commonly experience situations where healthcare professionals do not consider their need to understand and actively engage in questioning, discussion and information-seeking. This corresponds to the findings of several previous studies [12, 19-21]. The reason for physicians' and other professionals' neglect of the older persons' needs in this context may be an overestimation of the person's understanding of the post-discharge treatment plan and assumption that a person knows more about treatment and recovery than they actually do [44]. This emphasises the need for improved communication between professionals and older persons, as well as giving older persons the opportunity to be involved in decision making regarding their own health, to be standard practice.

Previous research shows that improved doctor-patient communication and patient participation can increase patient and provider satisfaction, as well as improve the patient's management of their chronic illness (es) [45]. Studies have demonstrated a correlation between effective physician-patient communication and improved health outcomes [46]. At the healthcare-system level, patient participation may potentially reduce healthcare costs [47], as well as medical errors [48]. Kristiansen et al. [49] found that being in control, experiencing a sense of power, feeling trust and being given the opportunity to participate was important for older persons' satisfaction with their care services.

All of this corresponds with strategies for increased user involvement that have appeared in the policy and action agendas of healthcare providers in recent years $[50,51]$. However, older persons may find it a challenge 
to be involved and participate actively in discharge planning because they find it difficult to understand what is being discussed or fail to feel included in the conversation [20]. To be able to involve older persons in the decision making, it is important that verbal and written communication and information is clear and easy to understand. Healthcare personnel need to take into account that the geriatric population are at risk of inadequate or marginal health literacy [52], and they should assess whether the information is understood by, and even understandable to, the person [53]. In addition, cognitive impairments or physical disabilities may interfere with older persons' ability to be their own advocates. Hence, healthcare delivery systems need to be carefully thought out in order to appropriately support these persons [54]. Some persons may prefer to have a limited involvement in decision making $[55,56]$, but in this case, it is important for healthcare providers to attempt to clarify the reason for this and try to find and encourage a level of involvement that is satisfactory to the patient.

Furthermore, a study by Richardson et al. [57] found that persons aged 80 and over were reluctant to say or ask anything that could be perceived as criticising or complaining about the hospital or the hospital staff. This may be explained by the fact that this generation of older people has often had a lifelong experience with a paternalistic healthcare system [58], which aligns with our findings. To reduce the feelings of disempowerment and improve their experience of the transitional process, better bidirectional communication and information is needed. Professionals will need to modify and/or bolster their interviewing skills, as well as provided oral and written information, and spend additional time getting to know the older person and determine their values, goals and preferences [21].

Our findings are in line with previous studies that report that healthcare sectors experience difficulties in communication and systematic information exchange, as well as with coordinating, which could lead to adverse events, such as readmissions, drug events, and falls [26]. The multifaceted arrangements of multiple service providers and the complexity of the needs of older patients make coordination of care challenging [59].

The participants in these studies were generally eager to go home and, they stressed their ability to manage on their own at home after discharge. This corresponds to a previous study by Ebrahimi et al. [60], which found that older persons made great efforts to find ways to master life's new situation, be useful and not be a burden to others. However, inadequate assessment of personal needs evoked feelings of frustration and led to limited social interactions, social isolation and loneliness. The findings indicate that when environmental and psychosocial needs are unaddressed, it affects self-management and recovery at home after discharge. Involving informal caregivers in the discharge process and the assessment of the older persons' needs at home can give older people better opportunities to master life at home after hospital discharge, thus reducing the strain on family caregivers. The systematic review by Bauer et al. [29] revealed that conducting comprehensive discharge planning that includes the older person and their informal caregivers is directly related to a reduction in hospital readmission, shorter hospital stays and improved satisfaction with the healthcare experience.

\section{Implications for practice}

Given that most healthcare systems are moving towards a model that favours early discharge from hospital to home or community care, it is imperative to understand how care providers can improve continuity of care to make the transitional process smoother for older persons. Strategies should include:

- Older persons receiving (sufficient) information about their illness (es) and the course of the illness, medication, rehabilitation and psychosocial aspects of their recovery.

- Hospital discharge should include an assessment of the person's post-discharge needs when it comes to food, specialised equipment, transportation to follow-up appointments and general care at home. These identified needs should be communicated to the next level of care.

- Adapting verbal and written communication in order to make the information clearer and easy to understand. Healthcare professionals should also assess whether the information is understood by the patient.

- Incorporating older persons' involvement and perspectives into the decision-making process.

- Increasing the involvement of informal caregivers in the care planning.

- Strategies to reduce feelings of loneliness and social isolation after hospital discharge and resettling into the home situation.

- Better communication between service providers, professionals, older persons and their informal caregivers.

\section{Strengths and limitations of the study}

A major strength of this work is the application of a rigorous and systematic metasummary technique. Synthesising qualitative research is viewed as essential to achieving the goal of evidence-based practice, namely to use the best available evidence as the foundation for practice without methodological prejudice [31]. Another strength is that even though the included studies are from different 
countries with variously structured healthcare systems, there was considerable agreement across the research about how older persons experienced hospital discharge and adapting to daily life at home afterwards.

Because care models are continuously reformed-for example, the Affordable Care Act in the United States [61], evolving models of ambulatory and sub-acute care funded by the Australian government [9] and evolving models in the British Healthcare Trusts [62] - we only included studies published between 2006 and 2017 to ensure the findings would be relevant to current clinical practice. These studies are grounded in policy and practice contexts of the more recent transitional care and integrated care models in the countries represented. However, by excluding earlier studies, our study may not address issues pertaining to user experience in discharge and care transitions from preceding healthcare arrangements. We also only included studies published in English in our review; therefore, we may have missed relevant research from non-English-speaking countries.

\section{Conclusions}

This study contributes to our understanding of older persons' experiences of the transition from hospital to home and may provide an important frame for understanding and improving older persons' satisfaction with the healthcare services provided in transitional care. The results emphasise the importance of assessment and planning, information and education, preparation of the home environment, the involvement of the older person and caregivers and supporting self-management in the discharge and follow-up care at home. Health professionals should actively cultivate their communication skills with an awareness of older persons' experiences, priorities and goals in order to provide healthcare focused on what is most relevant to older persons in transitional care situations. Better communication between older persons, hospital providers and home care providers is needed to improve coordination of care and facilitate recovery at home. The organisational structure may need to be redefined and reorganised to secure continuity of care and wellbeing of older persons in transitional care situations.

\section{Additional file}

Additional file 1: Table S1. Examples of participants' quotations

illustrating the results. (PDF $103 \mathrm{~kb}$ )

\section{Abbreviations}

HRQOL: Health-Related Quality of Life; JBI-QARI: Johanna Briggs Institute Qualitative Assessment and Review Instrument

\section{Acknowledgements}

The authors would like to thank librarian Malene W. Gundersen for her help and advice regarding the literature search.

\section{Funding}

The Research Council of Norway funded the salary of the first author but had no other participation in the project. This study is part of a larger project, titled Crosscare-Old (project.nr. 256644//H10).

Availability of data and materials

Data sharing is not applicable to this article, as no datasets were generated or analysed during the current study.

\section{Authors' contributions}

The first author $(\mathrm{CHH})$ conducted the literature search and initial screening for relevant studies. All authors conducted the selection of studies, quality appraisal and synthesising of data. $\mathrm{CHH}$ prepared the manuscript. All authors contributed to the development of the manuscript and approved the final manuscript.

Ethics approval and consent to participate

Not applicable.

Consent for publication

Not applicable.

\section{Competing interests}

The authors declare that they have no competing interests.

\section{Publisher's Note}

Springer Nature remains neutral with regard to jurisdictional claims in published maps and institutional affiliations.

\section{Author details}

${ }^{1}$ Department of Physiotherapy, Faculty of Health Sciences, OsloMet - Oslo Metropolitan University, Oslo, Norway. ${ }^{2}$ Department of Nursing and Health Promotion, Faculty of Health Sciences, OsloMet - Oslo Metropolitan University, Oslo, Norway. ${ }^{3}$ Bjørknes University College, Lovisenberggata 13, 0456 Oslo, Norway. ${ }^{4}$ European Palliative Care Research Centre (PRC), Department of Oncology, Oslo University Hospital and Institute of Clinical Medicine, University of Oslo, Oslo, Norway.

Received: 1 November 2017 Accepted: 24 March 2019

Published online: 11 April 2019

\section{References}

1. Coleman EA, Smith JD, Frank JC, Eilertsen TB, Thiare JN, Kramer AM. Development and testing of a measure designed to assess the quality of care transitions. Int J Integr Care. 2002;2:e02.

2. Volpato S, Onder G, Cavalieri M, Guerra G, Sioulis F, Maraldi C, Zuliani G, Fellin R. Italian Group of Pharmacoepidemiology in the elderly. Characteristics of nondisabled older patients developing new disability associated with medical illnesses and hospitalization. J Gen Intern Med. 2007;22(5):668-74

3. Haines TP, Russell T, Brauer SG, Erwin S, Lane P, Urry S, Jasiewicz J, Condie P. Effectiveness of a video-based exercise programme to reduce falls and improve health-related quality of life among older adults discharged from hospital: a pilot randomized controlled trial. Clin Rehabil. 2009;23(11):973-85.

4. Hofhuis JG, Spronk PE, van Stel HF, Schrijvers GJ, Rommes JH, Bakker J. The impact of critical illness on perceived health-related quality of life during ICU treatment, hospital stay, and after hospital discharge: a long-term follow-up study. Chest. 2008:133(2):377-85.

5. Mckeown $F$. The experiences of older people on discharge from hospital following assessment by the public health nurse. J Clin Nurs. 2007;16(3):469-76.

6. Forster AJ, Rose NGW, Van Walraven C, Stiell I. Adverse events following an emergency department visit. Qual Saf Health Care. 2007;16(1):17-22.

7. Thorslund M, Silverstein M. Care for older adults in the welfare state: theories, policies and realities. In: Bengtson VL, Gans D, Putney NM, Silverstein $M$, editors. Handbook of theories of aging. 2nd ed. New York: Springer; 2009. p. 629-39.

8. AARP Public Policy Institute. Aging in place: a state survey of livability policies and practices. https://assets.aarp.org/rgcenter/ppi/liv-com/aging-inplace-2011-full.pdf (2011). Accessed 5. July 2017. 
9. Australian Government. Caring for older Australians. In: Productivity comission. http://www.pc.gov.au/inquiries/completed/aged-care (2011). Accessed 8 Aug 2017.

10. Mansah M, Fernandez R, Griffiths R, Chang E. Effectiveness of strategies to promote safe transition of elderly people across care settings. JBI Libr Syst Rev. 2009:7(24):1036-90.

11. Perry MA, Hudson S, Ardis K. 'If I didn't have anybody, what would I have done?': Experiences of older adults and their discharge home after lower limb orthopaedic surgery. J Rehabil Med. 2011;43(10):916-22.

12. Rustad EC, Furnes B, Cronfalk BS, Dysvik E. Older patients' experiences during care transition. Patient Prefer Adherence. 2016;10:769-79.

13. Dossa A, Bokhour B, Hoenig H. Care transitions from the hospital to home for patients with mobility impairments: patient and family caregiver experiences. Rehabil Nurs. 2012;37(6):277-85

14. Knight DA, Thompson D, Mathie E, Dickinson A. 'Seamless care? Just a list would have helped!' Older people and their carer's experiences of support with medication on discharge home from hospital. Health Expect. 2013;16(3):277-91.

15. Neiterman E, Wodchis WP, Bourgeault IL. Experiences of older adults in transition from hospital to community. Can J Aging. 2015;34(1):90-9.

16. Rennke S, Ranji SR. Transitional care strategies from hospital to home: a review for the neurohospitalist. Neurohospitalist. 2015;5(1):35-42.

17. Mesteig M, Helbostad JL, Sletvold O, Rosstad T, Saltvedt I. Unwanted incidents during transition of geriatric patients from hospital to home: a prospective observational study. BMC Health Serv Res. 2010;10:1

18. Ekdahl AW, Hellstrom I, Andersson L, Friedrichsen M. Too complex and time-consuming to fit in! Physicians' experiences of elderly patients and their participation in medical decision making: a grounded theory study. BMJ Open. 2012;2(3):1-7. https://www.ncbi.nlm.nih.gov/pubmed/22654092.

19. Foss C, Hofoss D. Elderly persons' experiences of participation in hospital discharge process. Patient Educ Couns. 2011;85(1):68-73.

20. Efraimsson E, Sandman P-O, Rasmussen BH. 'They were talking about me' elderly women's experiences of taking part in a discharge planning conference. Scand J Caring Sci. 2006;20(1):68-78.

21. Bynum JP, Barre L, Reed C, Passow H. Participation of very old adults in healthcare decisions. Med Decis Mak. 2014;34(2):216-30.

22. Halvorsen K, Eide HK, Sortland K, Almendingen K. Documentation and communication of nutritional care for elderly hospitalized patients: perspectives of nurses and undergraduate nurses in hospitals and nursing homes. BMC Nurs. 2016;15:70.

23. Bull MJ, Roberts J. Components of a proper hospital discharge for elders. J Adv Nurs. 2001;35(4):571-81.

24. Almborg AH, Ulander K, Thulin A, Berg S. Patients' perceptions of their participation in discharge planning after acute stroke. J Clin Nurs. 2009; 18(2):199-209.

25. Birmingham J. Discharge planning: a collaboration between provider and payer case managers using Medicare's conditions of participation. Lippincotts Case Manag. 2004;9(3):147-51.

26. Laugaland K, Aase K, Barach P. Interventions to improve patient safety in transitional care-a review of the evidence. Work. 2012;41(Suppl 1):2915-24.

27. Allen J, Hutchinson AM, Brown R, Livingston PM. Quality care outcomes following transitional care interventions for older people from hospital to home: a systematic review. BMC Health Serv Res. 2014;14:346.

28. Mistiaen $\mathrm{P}$, Francke AL, Poot E. Interventions aimed at reducing problems in adult patients discharged from hospital to home: a systematic meta-review. BMC Health Serv Res. 2007;7:47.

29. Bauer M, Fitzgerald L, Haesler E, Manfrin M. Hospital discharge planning for frail older people and their family. Are we delivering best practice? A review of the evidence. J Clin Nurs. 2009;18(18):2539-46

30. Andreasen J, Lund $H$, Aadahl M, Sorensen EE. The experience of daily life of acutely admitted frail elderly patients one week after discharge from the hospital. Int J Qual Stud Health Well-being. 2015;10:27370.

31. Sandelowski M, Barroso J. Handbook for synthesizing qualitative research. New York: Springer; 2007.

32. Joanna Briggs Institute. JBI Critical Appraisal Checklist for Qualitative Research. In: Institut TJB, editors. http://joannabriggs.org/assets/docs/criticalappraisal-tools/JBI_Critical_Appraisal-Checklist_for_Qualitative_Research2017. pdf (2017). Accessed 8 Mar 2018.

33. Lewin S, Glenton C, Munthe-Kaas H, Carlsen B, Colvin CJ, Gulmezoglu M, Noyes J, Booth A, Garside R, Rashidian A. Using qualitative evidence in decision making for health and social interventions: an approach to assess confidence in findings from qualitative evidence syntheses (GRADE-CERQual). PLoS Med. 2015;12(10): e1001895.

34. Creswell JW, Poth CN. Qualitative inquiry \& research design: choosing among five approaches. 4th ed. SAGE: Los Angeles; 2018.

35. Waibel S, Henao D, Aller MB, Vargas I, Vazquez ML. What do we know about patients' perceptions of continuity of care? A meta-synthesis of qualitative studies. Int J Qual Health Care. 2012;24(1):39-48.

36. Allen J, Hutchinson AM, Brown R, Livingston APM. User experience and care integration in transitional care for older people from hospital to home: A metasynthesis. Qual Health Res. 2017;27(1):24-36.

37. Slatyer S, Toye C, Popescu A, Young J, Matthews A, Hill A, Williamson D. Early representation to hospital after discharge from an acute medical unit: perspectives of older patients, their family caregivers and health professionals. J Clin Nurs. 2013;22(3-4):445-55.

38. Jones GB. Older persons' experiences of hospital patient education and selfmanagement of their chronic diseases once discharged home. Dissertation Abstracts International: Section B: The Sciences and Engineering. 2016;77(2-B [E]).

39. Reay PA, Horner B, Duggan R. The patient's experience of early discharge following total hip replacement. Int J Orthop Trauma Nurs. 2015;19(3):131-9.

40. Dilworth S, Higgins I, Parker V. Feeling let down: an exploratory study of the experiences of older people who were readmitted to hospital following a recent discharge. Contemp Nurse. 2012;42(2):280-8.

41. Bagge M, Norris P, Heydon S, Tordoff J. Older people's experiences of medicine changes on leaving hospital. RSAP. 2014;10(5):791-800.

42. Rydeman I, Tornkvist L. Getting prepared for life at home in the discharge process-from the perspective of the older persons and their relatives. Int J Older People Nursing. 2010;5(4):254-64.

43. Karlsson V, Bergbom I, Ringdal M, Jonsson A. After discharge home: a qualitative analysis of older ICU patients' experiences and care needs. Scand J Caring Sci. 2016;30(4):749-56.

44. Calkins DR, Davis RB, Reiley P, Phillips RS, Pineo KL, Delbanco TL, lezzoni LI. Patient-physician communication at hospital discharge and patients understanding of the postdischarge treatment plan. Arch Intern Med. 1997; 157(9):1026-30.

45. Mavis B, Holmes Rovner M, Jorgenson S, Coffey J, Anand N, Bulica E, Gaulden CM, Peacock J, Ernst A. Patient participation in clinical encounters: a systematic review to identify self-report measures. Health Expect. 2015;18(6):1827-43.

46. Stewart MA. Effective physician-patient communication and health outcomes: a review. CMAJ. 1995;152(9):1423-33.

47. O'Connor AM, Wennberg JE, Legare F, Llewellyn-Thomas HA, Moulton BW, Sepucha KR, Sodano AG, King JS. Toward the 'tipping point': decision aids and informed patient choice. Health Aff (Millwood). 2007; 26(3):716-25.

48. Hibbard JH, Peters E, Slovic P, Tusler M. Can patients be part of the solution? Views on their role in preventing medical errors. Med Care Res Rev. 2005;62(5): 601-16.

49. Kristensson J, Hallberg IR, Ekwall AK. Frail older adults' experiences of receiving healthcare and social services. J Gerontol Nurs. 2010;36(10):20-8 quiz 30-21.

50. Transforming participation in NHS England: Transforming Participation in Health and Care, Guidance for Commissioners. https://www.england.nhs.uk 2013/09/trans-part/ (2013). Accessed 11 Aug 2017.

51. Government of Norway. The Coordination Reform. Ministry of Health and Care Services; 2009. Report No:: 47 to the Storting (2008-2009). https://www. regjeringen.no/contentassets/d4f0e16ad32e4bbd8d8ab5c21445a5dd/en-gb/pdfs/ stm200820090047000en_pdfs.pdf (2009). Accessed 2. Aug 2017.

52. Pelikan JM, Röthlin F, Ganahl K. Comparative report of health literacy in eight EU member states. The HLS-EU Consortium; 2012.

53. Hesselink G, Zegers M, Vernooij-Dassen M, Barach P, Kalkman C, Flink M, Ohlen G, Olsson M, Bergenbrant S, Orrego C, et al. Improving patient discharge and reducing hospital readmissions by using intervention mapping. BMC Health Serv Res. 2014:14:389.

54. Coleman EA. Falling through the cracks: challenges and opportunities for improving transitional care for persons with continuous complex care needs. J Am Geriatr Soc. 2003;51(4):549-55.

55. Belcher VN, Fried TR, Agostini JV, Tinetti ME. Views of older adults on patient participation in medication-related decision making. J Gen Intern Med. 2006;21(4): 298-303.

56. Ekdahl AW, Andersson L, Friedrichsen M. 'They do what they think is the best for me.' Frail elderly patients' preferences for participation in their care during hospitalization. Patient Educ Couns 2010;80(2):233-240. 
57. Richardson S, Casey M, Hider P. Following the patient journey: older persons' experiences of emergency departments and discharge. Accid Emerg Nursing. 2007;15(3):134-40.

58. Bridges J, Flatley M, Meyer J. Older people's and relatives' experiences in acute care settings: systematic review and synthesis of qualitative studies. Int J Nurs Stud. 2010;47(1):89-107.

59. Ham C, Imison C, Goodwin N, Dixon A, South P. Where next for the NHS reforms? The case for integrated care. London: The Kings Fund; 2011. https://www.kingsfund.org.uk/sites/default/files/where-next-nhs-reformscase-for-integrated-care-ham-imison-goodwin-dixon-south-kings-fund-may2011.pdf. Accessed 1 Aug 2017

60. Ebrahimi Z, Wilhelmson K, Moore CD, Jakobsson A. Frail elders' experiences with and perceptions of health. Qual Health Res. 2012;22(11):1513-23.

61. Healthcare. Office of the Legislative Counsel Gov. Patient Protection and Affordable Care Act Health-Related Portions of the Health Care and Education Reconciliation Act of 2010. 2010. http://housedocs.house.gov/ energycommerce/ppacacon.pdf. Accessed 4 Mar 2019.

62. Lewis RQ, Rosen R, Goodwin N, Dixon J. Where next for integrated care organisations in the English NHS? The Nuffield Trust; https://www. nuffieldtrust.org.uk/files/2017-01/where-next-integrated-care-english-nhsweb-final.pdf (2010). Accessed 25 July 2018.

Ready to submit your research? Choose BMC and benefit from:

- fast, convenient online submission

- thorough peer review by experienced researchers in your field

- rapid publication on acceptance

- support for research data, including large and complex data types

- gold Open Access which fosters wider collaboration and increased citations

- maximum visibility for your research: over $100 \mathrm{M}$ website views per year

At $\mathrm{BMC}$, research is always in progress.

Learn more biomedcentral.com/submissions 